\title{
Subgrid-scale model for large-eddy simulation of transition and turbulence in compressible flows
}

Cite as: Phys. Fluids 31, 125118 (2019); https://doi.org/10.1063/1.5128061

Submitted: 17 September 2019 . Accepted: 30 November 2019 . Published Online: 20 December 2019

Hao Zhou (周浩), Xinliang Li (李新亮), Han Qi (齐涵), and Changping Yu (于长平) (D)

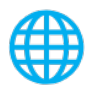

\section{ARTICLES YOU MAY BE INTERESTED IN}

Wall-modeled large-eddy simulations of spanwise rotating turbulent channels-Comparing a physics-based approach and a data-based approach

Physics of Fluids 31, 125105 (2019); https://doi.org/10.1063/1.5129178

Solid wall and open boundary conditions in hybrid recursive regularized lattice Boltzmann method for compressible flows

Physics of Fluids 31, 126103 (2019); https://doi.org/10.1063/1.5129138

Flow structures in transitional and turbulent boundary layers

Physics of Fluids 31, 111301 (2019); https://doi.org/10.1063/1.5121810

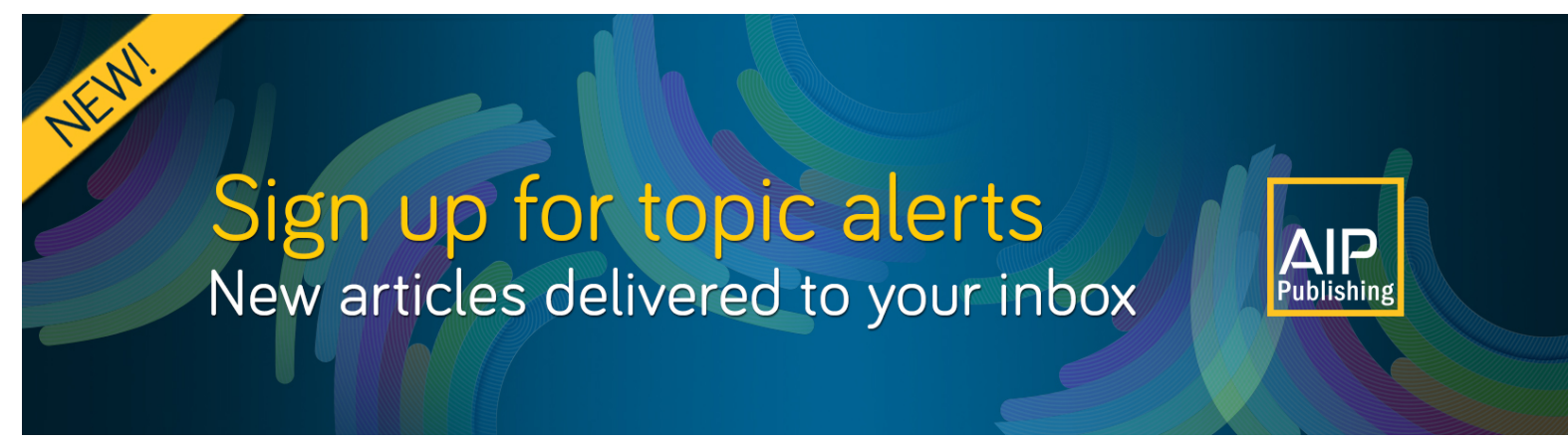




\title{
Subgrid-scale model for large-eddy simulation of transition and turbulence in compressible flows
}

\author{
Cite as: Phys. Fluids 31, 125118 (2019); doi: 10.1063/1.5128061 \\ Submitted: 17 September 2019 - Accepted: 30 November 2019 • \\ Published Online: 20 December 2019
}

Hao Zhou (周浩), ${ }^{1,2}$ Xinliang Li (李新亮), ${ }^{1,2}$ Han Qi (齐涵), ${ }^{1,2}$ and Changping Yu (于长平) ${ }^{1,2, a)}$ (D)

\author{
AFFILIATIONS \\ ${ }^{1}$ LHD, Institute of Mechanics, Chinese Academy of Sciences, Beijing 100190, China \\ ${ }^{2}$ School of Engineering Science, University of Chinese Academy of Sciences, Beijing 100049, China
}

a) Electronic mail: cpyu@imech.ac.cn

\begin{abstract}
This article focuses on a subgrid-scale (SGS) eddy viscosity model based on helicity which is derived from our previous research [Yu et al., "Subgrid-scale eddy viscosity model for helical turbulence," Phys. Fluids 25, 095101 (2013)] for large-eddy simulation of transition and turbulence in compressible flows. Based on the character of the compressible boundary layer over a flat plate, we obtain from theoretical analysis that this model can automatically distinguish laminar flow and turbulence and can also simulate turbulence well. Meanwhile, an $a$ priori test using direct numerical simulation (DNS) data of a spatially developing flat-plate boundary layer at $M a=2.25$ shows that the helicity model can clearly differentiate laminar, transitional, and turbulent regions. Comparing the numerical simulation results with DNS and other SGS models in the spatially developing boundary-layer over a flat plate, we find that the suggested model could precisely predict the onset of transition, transition peak, skin-friction coefficient, mean velocity profile, mean temperature profile, and turbulence intensities. In the case of a compression ramp, the model can well simulate the bypass-type transition, the separated and reattached points, and the size of the separation bubble in the corner region. Furthermore, the prominent advantage of the proposed model can predict transitional flow exactly with no explicit filtering or dynamic procedure.
\end{abstract}

Published under license by AIP Publishing. https://doi.org/10.1063/1.5128061

\section{INTRODUCTION}

Transition to turbulent flows exists widely in nature and engineering machinery, particularly in turbomachinery and flying vehicles. Large eddy simulation (LES) is an important method to correctly predict the transition point and transition process.

Since Smagorinsky presented the first subgrid-scale (SGS) model for large-eddy simulation (LES) in the 1970s, ${ }^{1}$ the subgridscale (SGS) models and LES methods have gained great success and development. Similar to the Smagorinsky model (SM), the most commonly used SGS model is the eddy-viscosity model, and various other forms of SGS eddy-viscosity models have also been proposed so far. Using the second-order velocity structure function, Métais and Lesieur ${ }^{2}$ proposed the second-order structure function model, which is a generalization of the spectral eddy viscosity model in physical space. Similarly, Cui et al. ${ }^{3}$ took the second- and third-order structure function to develop the CZZS model. The wall-adapting local eddy-viscosity (WALE) model was proposed by Nicoud and Ducros, ${ }^{4}$ which is based on the square of the velocity gradient tensor, and its SGS eddy viscosity could recover the proper $y^{3}$ near-wall scaling without the dynamic procedure. Vreman ${ }^{5}$ suggested a SGS model for shear flows which has a simple formula and relatively small dissipation in the transitional and near-wall regions. Recently, some nonlinear eddy viscosity models have also been proposed to simulate some special turbulent flows, such as rotating turbulence and wall-bounded turbulence. ${ }^{6,7}$ Most of the SGS models are proposed on account of kinetic energy of turbulence. Besides the SGS models, some practical LES methods have also been suggested to ensure that the SGS model simulates turbulence better. ${ }^{8}$ Germano et al. ${ }^{9}$ suggested the dynamic procedure for the SGS models, which is referred to as the Germano identity and has become the basis of various dynamic models. ${ }^{10-14}$ For dynamic SGS models, the local 
model coefficient in the turbulent flow can be adjusted dynamically with the simulation process. Some additional transport equations, such as the SGS turbulent kinetic energy transport equation, were applied to adjust the coefficient of the SGS model in LES of complex turbulence. ${ }^{15}$ These modifications are used to remedy the lack of localization of the information and to adapt the model better to the local state of the flow.

During the transition process, the flow parameters such as the skin-friction and heat flux will increase rapidly from the laminar status to the peak of transition status. Therefore, correctly simulating the length of the surface where the boundary layer is laminar might be necessary for overall drag prediction. ${ }^{16}$ Horiuti $^{17}$ used the constant coefficient Smagorinsky model (CSM) to simulate a transitional channel flow and found that the CSM provides excessive dissipation in the transition process, which would impede transition to occur in the flow. Ducros et al. reformulated the structure-function model to simulate the transition in a spatial boundary layer at $M a=0.5$, and they got an acceptable result. ${ }^{18}$ Huai et al. applied the dynamic Smagorinsky model (DSM) to simulate a transitional flat-plate boundary layer for the first time, and the simulation results show that DSM could predict transition. ${ }^{19}$ More recently, Sayadi and Moin assessed the capabilities of several existing SGS models, such as the CSM and Vreman model, the dynamic mixed scale similarity model, and the dynamic one-equation kinetic energy model to predict the transition point and skin-friction all around the transition process, and the results show that the CSM and constant coefficient Vreman model cannot capture the transition location, and the dynamic SGS models could estimate the transition point correctly if the grid resolution is fine enough. ${ }^{16}$

In this article, using the boundary layer over a flat plate, we analyze the helicity model (HM) deriving from our previous research ${ }^{20}$ and find that $\mathrm{HM}$ can predict transition process in theory. Through a priori and a posteriori tests in the compressible boundary layer over a flat plate and the supersonic turbulent boundary layer over a compression ramp, the HM is accurately verified to simulate transition to turbulence. We introduce the model and generalize its validity to transitional flow theoretically in Sec. II. A priori and a posteriori tests are performed in Secs. III and IV, respectively.

\section{ANALYSIS IN THEORY}

For compressible turbulent flows, the filtered Navier-Stokes equations of LES can be written as follows:

$$
\begin{gathered}
\frac{\partial \bar{\rho}}{\partial t}+\frac{\partial \bar{\rho} \tilde{u}_{i}}{\partial x_{i}}=0, \\
\frac{\partial \bar{\rho} \tilde{u}_{i}}{\partial t}+\frac{\partial \bar{\rho} \tilde{u}_{i} \tilde{u}_{j}}{\partial x_{j}}=-\frac{\partial}{\partial x_{j}}\left(\bar{p} \delta_{i j}-\tilde{\sigma}_{i j}+\tau_{i j}\right), \\
\frac{\partial \bar{\rho} \tilde{E}}{\partial t}+\frac{\partial(\bar{\rho} \tilde{E}+\bar{p}) \tilde{u}_{j}}{\partial x_{j}}=-\frac{\partial}{\partial x_{j}}\left(\tilde{q}_{j}-\tilde{\sigma}_{i j} \tilde{u}_{i}+J_{j}+Q_{j}\right), \\
\bar{p}=\bar{\rho} R \tilde{T},
\end{gathered}
$$

where a bar denotes spatial filtering at scale $\Delta$ using a smooth lowpass filter function $G_{\Delta}(\mathbf{r})$ [e.g., $\bar{\rho}(x)=\int G_{\Delta}(\mathbf{r}) \rho(x+r) d \mathbf{r}$ represents the resolved density field] and a tilde denotes spatial Favre filtering as $\tilde{\phi}=\frac{\overline{\rho \phi}}{\bar{\rho}}$.

In (1)-(4), $\rho, u_{i}, T, E$, and $R$ denote density, velocity, temperature, total energy, and specific gas constant, respectively. The viscous stress tensor $\tilde{\sigma}_{i j}$ and the heat flux vector $\tilde{q}_{j}$ are given by the following equation:

$$
\begin{gathered}
\tilde{\sigma}_{i j}=2 \mu(\tilde{T})\left(\tilde{S}_{i j}-\frac{1}{3} \delta_{i j} \tilde{S}_{k k}\right), \\
\tilde{q}_{j}=\frac{C_{p} \mu(\tilde{T})}{\operatorname{Pr}} \frac{\partial \tilde{T}}{\partial x_{j}},
\end{gathered}
$$

where $C_{p}, P r$ are the specific heat at constant pressure and molecular Prandtl number, $\mu=\frac{1}{R e} \frac{\tilde{T}^{3 / 2}\left(1+T_{s} / \tilde{T}_{\infty}\right)}{\tilde{T}+T_{s} / \tilde{T}_{\infty}}$ is the molecular viscosity calculated using Sutherland's law for given $T_{s}=110.3 \mathrm{~K}$, $R e=\rho_{\infty} U_{\infty} L / \mu_{\infty}$ is the Reynolds number, and $\tilde{S}_{i j}=\frac{1}{2}\left(\frac{\partial \tilde{u}_{i}}{\partial x_{j}}+\frac{\partial \tilde{u}_{j}}{\partial x_{i}}\right)$ is the resolved strain-rate tensor.

In (1)-(4), there are some unclosed terms: the SGS stress tensor:

$$
\tau_{i j}=\bar{\rho}\left(\widetilde{u_{i} u_{j}}-\tilde{u}_{i} \tilde{u}_{j}\right)
$$

the SGS heat flux:

$$
Q_{j}=\bar{\rho} C_{p}\left(\widetilde{u_{j} T}-\tilde{u}_{j} \tilde{T}\right),
$$

and the SGS turbulent diffusion

$$
J_{j}=\frac{1}{2}\left(\bar{\rho} \widetilde{u_{j} u_{i} u_{i}}-\bar{\rho} \tilde{u}_{j} \widetilde{u_{i} u_{i}}\right) .
$$

It is suggested that the SGS turbulent diffusion can be approximated as $J_{j}=\tau_{i j} \tilde{u}_{i}{ }^{21}$ The SGS stress tensor $\tau_{i j}$ and the SGS heat flux $Q_{j}$ need to be modeled based on the resolved quantities. Most SGS models are based on eddy-viscosity assumption, and the form of SGS stress tensor $\tau_{i j}$ and the SGS heat flux $Q_{j}$ are given by the following equation:

$$
\begin{aligned}
\tau_{i j}^{\text {mod }}-\frac{1}{3} \tau_{k k}^{s g s} \delta_{i j} & =-2 \mu_{s g s}\left(\tilde{S}_{i j}-\frac{1}{3} \tilde{S}_{k k} \delta_{i j}\right), \\
Q_{j}^{\text {mod }} & =-\frac{C_{p} \mu_{s g s}}{P r_{t}} \frac{\partial \tilde{T}}{\partial x_{j}}
\end{aligned}
$$

where $\mu_{\text {sgs }}$ is SGS viscosity, and $P r_{t}$ is the SGS Prandtl number.

The often used SGS model is the Smagorinsky model,

$$
\begin{aligned}
& \mu_{\text {sgs }}=\bar{\rho}\left(C_{s} \Delta\right)^{2}|\tilde{S}|, \\
& \tau_{k k}^{s g s}=2 C_{I} \bar{\rho} \Delta^{2}|\tilde{S}|^{2},
\end{aligned}
$$

where $|\tilde{S}|=\sqrt{\left(2 \tilde{S}_{i j} \tilde{S}_{i j}\right)}$ is the magnitude of resolved strain tensor, and $C_{s}$ and $C_{I}$ are the model coefficients.

In our previous research, we proposed a novel eddy-viscosity model based on helicity in full turbulent flow, ${ }^{20}$ which is distinguished from the traditional SGS eddy-viscosity model based on kinetic energy. The new SGS model can be called helicity model (HM) here, and the SGS eddy viscosity of the anisotropic part of the SGS tensor is modeled as follows:

$$
\mu_{s g s}=\bar{\rho} C_{r} \Delta^{5 / 2} \tilde{S}_{s r}
$$


To secure the isotropic part of the SGS tensor $\tau_{k k}^{s g s}$ keeping the closed status in strong compressible laminar flows, we modified the isotropic part of the SGS tensor as follows:

$$
\tau_{k k}^{s g s}=2 \bar{\rho} C_{i r} \Delta^{3} \tilde{S}_{s r}^{2},
$$

Thus, the helicity model for SGS stress and SGS heat flux can be generalized as follows:

$$
\tau_{i j}^{\text {mod }}=-2 \bar{\rho} C_{r} \Delta^{5 / 2} \tilde{S}_{s r}\left(\tilde{S}_{i j}-\frac{1}{3} \tilde{S}_{k k} \delta_{i j}\right)+\frac{2}{3} \bar{\rho} C_{i r} \Delta^{3} \tilde{S}_{s r}^{2} \delta_{i j},
$$

and

$$
Q_{j}^{m o d}=-C_{r} \frac{C_{p} \bar{\rho} \Delta^{5 / 2} \tilde{S}_{s r}}{P r_{t}} \frac{\partial \tilde{T}}{\partial x_{j}} .
$$

In (14)-(17), $\tilde{S}_{s r}=\left|2 \tilde{S}_{i j} \tilde{R}_{i j}\right|^{1 / 2}$ and $\tilde{R}_{i j}=\frac{1}{2}\left(\partial_{j} \tilde{\omega}_{i}+\partial_{i} \tilde{\omega}_{j}\right)$ are the symmetric parts of the resolved vorticity $\left(\tilde{\omega}_{i}\right)$ gradient tensor, and $C_{r}$ and $C_{i r}$ are the model coefficients.

The HM is shown to be proportional to the product of the resolved velocity strain rate tensor and the vorticity gradient tensor. The detailed derivation can be seen in the author's published literature. ${ }^{20}$ In order to better illustrate the rationality of the helicity model, we also prove the Galileo invariance of the model in Appendix A.

For transitional flow, as noted in Sec. I, CSM fails to predict the "point" of transition accurately, because CSM is still active in the laminar region and supplies excessive dissipation which will damp the development of disturbances. As a result, the transition does not occur in the boundary layer. ${ }^{22}$ The key to ensuring effectiveness of the SGS model in transition prediction is to differentiate the laminar region and turbulent region automatically. According to the previous experience, the most commonly used approach to solve this problem is the dynamic procedure. However, the dynamic procedure need test filtering, which is inconvenient to apply to the flow with complex boundaries. Through theoretical analysis, we find that the helicity model can be used to successfully simulate transitional flow directly.

For a wall-bounded laminar flow (e.g., a boundary layer over a flat plate), the laminar boundary layer equations can be approximately regarded as two-dimensional equations. For simplicity (spanwise direction velocity $w \approx 0$ ), the compressible laminar boundary layer equations are chosen to analyze. In the equations, $x$ indicates the streamwise direction and $y$ indicates the wall-normal direction. The continuity equation and the momentum equation can be expressed as follows:

$$
\begin{gathered}
\frac{\partial \rho}{\partial t}+\frac{\partial \rho u}{\partial x}+\frac{\partial \rho v}{\partial y}=0 \\
\frac{\partial \rho u}{\partial t}+\frac{\partial \rho u u}{\partial x}+\frac{\partial \rho u v}{\partial y}=-\frac{\partial p}{\partial x}+\frac{\partial \sigma_{11}}{\partial x}+\frac{\partial \sigma_{12}}{\partial y}, \\
\frac{\partial \rho v}{\partial t}+\frac{\partial \rho u v}{\partial x}+\frac{\partial \rho v v}{\partial y}=-\frac{\partial p}{\partial y}+\frac{\partial \sigma_{12}}{\partial x}+\frac{\partial \sigma_{22}}{\partial y} .
\end{gathered}
$$

The plate length scale $L$, free-stream density $\rho_{0}$, and free-stream velocity $U$ are used to define the nondimensional independent variables which are regarded as the characteristic length, characteristic density, and characteristic velocity, respectively. Then, in the boundary layer, if the scale of the $x$ direction is $x \sim L$, the scale of the $y$ direction is $y \sim \delta$, where $\delta$ is the boundary layer thickness, and the magnitude of the $x$-direction velocity is $\rho u \sim \rho_{0} U$, then the results are as follows:

$$
\frac{1}{\rho_{0}} \frac{\partial \rho u}{\partial x} \sim \frac{\partial \tilde{u}}{\partial x} \sim \frac{U}{L}, \quad \frac{1}{\rho_{0}} \frac{\partial \rho u}{\partial y} \sim \frac{\partial \tilde{u}}{\partial y} \sim \frac{U}{\delta} .
$$

In the compressible laminar boundary layer of the flat plate, when the flow was approximately assumed as quasi-steady flow in this case, the continuity Eq. (22) could be written as $\partial(\rho u) / \partial x+\partial(\rho v) / \partial y$ $=0$, and the magnitude of the two terms should be equivalent,

$$
\frac{\partial \tilde{v}}{\partial y} \sim \frac{1}{\rho_{0}} \frac{\partial \rho v}{\partial y} \sim\left|\frac{1}{\rho_{0}} \frac{\partial \rho u}{\partial x}\right| \sim \frac{U}{L} .
$$

Based on these relations, we obtain the following:

$$
\begin{gathered}
\tilde{v} \sim \frac{U}{L} \delta, \quad \frac{\partial \tilde{v}}{\partial x} \sim \frac{U \delta}{L^{2}}, \\
\tilde{\omega}_{x}=\frac{\partial \tilde{w}}{\partial y}-\frac{\partial \tilde{v}}{\partial z} \sim 0, \quad \tilde{\omega}_{y}=\frac{\partial \tilde{u}}{\partial z}-\frac{\partial \tilde{w}}{\partial x} \sim 0, \\
\tilde{\omega}_{z}=\frac{\partial \tilde{v}}{\partial x}-\frac{\partial \tilde{u}}{\partial y} \sim \frac{U}{\delta} .
\end{gathered}
$$

Consequently, the strain rate tensor $\tilde{S}_{i j}$ can be defined as follows:

$$
\tilde{S}_{i j}=\frac{1}{2}\left(\frac{\partial \tilde{u}_{i}}{\partial x_{j}}+\frac{\partial \tilde{u}_{j}}{\partial x_{i}}\right) \sim\left[\begin{array}{ccc}
\frac{U}{L} & \frac{U}{\delta} & 0 \\
\frac{U}{\delta} & \frac{U}{L} & 0 \\
0 & 0 & 0
\end{array}\right],
$$

and the symmetric part of the vorticity gradient tensor $\tilde{R}_{i j}$ is as follows:

$$
\tilde{R}_{i j}=\frac{1}{2}\left(\frac{\partial \tilde{\omega}_{i}}{\partial x_{j}}+\frac{\partial \tilde{\omega}_{j}}{\partial x_{i}}\right) \sim\left[\begin{array}{ccc}
0 & 0 & \frac{U}{L \delta} \\
0 & 0 & \frac{U}{\delta^{2}} \\
\frac{U}{L \delta} & \frac{U}{\delta^{2}} & 0
\end{array}\right] .
$$

Thus, we can get such relationships for $|\tilde{S}|$ and $\left|\tilde{S}_{s r}\right|$,

$$
|\tilde{S}| \sim \frac{U}{\delta}, \quad\left|\tilde{S}_{s r}\right| \sim 0 .
$$

For this dimension analysis, we can see that the SGS viscosity of the helicity model is just like a switch in the transition process, and it will remain closed in laminar states and gradually open during the transition process.

More generally, even in unsteady compressible laminar boundary layer flow over a flat plate, we can still obtain such a relation as the following one:

$$
\begin{aligned}
\tilde{S}_{i j} & =\left[\begin{array}{ccc}
\frac{\partial \tilde{u}}{\partial x} & \frac{1}{2}\left(\frac{\partial \tilde{u}}{\partial y}+\frac{\partial \tilde{v}}{\partial x}\right) & 0 \\
\frac{1}{2}\left(\frac{\partial \tilde{u}}{\partial y}+\frac{\partial \tilde{v}}{\partial x}\right) & \frac{\partial \tilde{v}}{\partial y} & 0 \\
0 & 0 & 0
\end{array}\right], \\
\tilde{R}_{i j} & =\left[\begin{array}{ccc}
0 & 0 & \frac{1}{2} \frac{\partial \tilde{\omega}_{z}}{\partial x} \\
0 & 0 & \frac{1}{2} \frac{\partial \tilde{\omega}_{z}}{\partial y} \\
\frac{1}{2} \frac{\partial \tilde{\omega}_{z}}{\partial x} & \frac{1}{2} \frac{\partial \tilde{\omega}_{z}}{\partial y} & 0
\end{array}\right] .
\end{aligned}
$$


Then,

$$
\begin{aligned}
|\tilde{S}| & =\left|2 \tilde{S}_{i j} \tilde{S}_{i j}\right|^{1 / 2} \\
& =\left[2\left(\frac{\partial \tilde{u}}{\partial x}\right)^{2}+\left(\frac{\partial \tilde{u}}{\partial y}+\frac{\partial \tilde{v}}{\partial x}\right)^{2}+2\left(\frac{\partial \tilde{v}}{\partial y}\right)^{2}\right]^{1 / 2} .
\end{aligned}
$$

Thus, in the compressible laminar boundary layer,

$$
|\tilde{S}|=\left|2 \tilde{S}_{i j} \tilde{S}_{i j}\right|^{1 / 2} \neq 0, \quad \tilde{S}_{s r}=\left|2 \tilde{S}_{i j} \tilde{R}_{i j}\right|^{1 / 2} \approx 0 .
$$

From the analysis above, we can find that the SGS viscosity of the Smagorinsky model is still a large value in the laminar region, and it will produce overdissipation, damping the disturbances to decay prior to the happening of transition. Therefore, the "point" of transition is not predicted accurately with CSM. On the contrary, the SGS viscosity of the helicity model is close to zero in the laminar region, and the SGS dissipation will be negligible in such a case which will guarantee the development of linear unstable waves and the occurrence of transition.

\section{A PRIORI TEST}

In this section, an a priori test will be presented for the SGS viscosity of the Smagorinsky model and the helicity model using direct numerical simulation (DNS) data of a supersonic spatially developing boundary layer ${ }^{23}$ over a flat plate with a free-stream Mach number of 2.25 and a Reynolds number $(R e)$ of 635000 . The DNS employs a seventh-order difference scheme for spatial discretization and a third-order Runge-Kutta method for time advancement. The "viscous derivatives" and viscous flux function are determined by a sixth-order difference scheme. The computational domain (see Fig. 1) is bounded by in-flow and out-flow boundaries, a wall boundary, a far-field boundary, and the two boundaries (periodic) in the spanwise direction. The computational domain used in this test has a size of $L_{x} \times L_{y} \times L_{z}=6 \times 0.3 \times 0.175$ in. in the streamwise, wall-normal, and spanwise directions. The DNS data are filtered in spanwise direction with top-hat filter and the filter width is $\bar{\Delta}=8 \Delta_{z}$, where $\Delta_{z}$ is mesh spacing of DNS gird in spanwise direction.

Figure 2(a) shows the normalized SGS viscosity of the Smagorinsky model and helicity model along the streamwise direction at $y^{+}=15$ as a function of the Reynolds number based on the conditions at the outer edge of the boundary layer $R e_{x}$. The black circle (defined) in Fig. 2(a) represents the defined SGS viscosity, which is obtained directly from its definition, as shown in Eq. (10). In the laminar region $\left(0<R e_{x}<4 \times 10^{-6}\right)$ and transitional region $\left(4 \times 10^{-6}<R e_{x}<4.8 \times 10^{-6}\right)$, we can see that the SGS viscosity of the Smagorinsky model $\left(\mu_{s g s}^{S M} / \mu\right)$ has a large magnitude in contrast to its value in the turbulent region, which is the main reason of producing large SGS dissipation in laminar and transitional regions. On the contrary, the SGS viscosity of the helicity model has
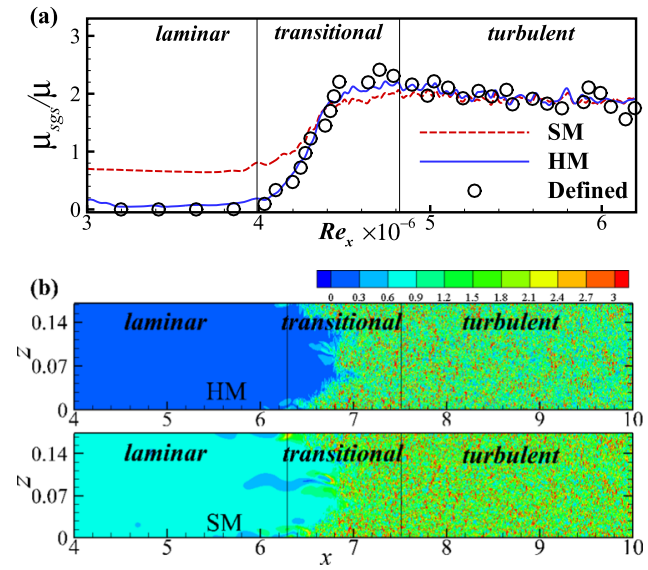

FIG. 2. (a) Distribution of normalized eddy viscosity $\left(\mu_{s g s} / \mu\right)$ of SM and HM at $y^{+}=15$ as a function of streamwise Reynolds number obtained a priori using DNS data. (b) Instantaneous contours of normalized eddy viscosity $\left(\mu_{\text {sgs }} / \mu\right)$ of SM and $H M$ in the $x-z$ plane at $y^{+} \approx 15 . \mu$ is molecular viscosity.

a negligible value the same as the modeled eddy viscosity in the laminar region, and at the same time, it can also provide a suitable SGS dissipation with the development of transition. In the turbulent region, they have the same behavior.

We display in Fig. 2(b) the instantaneous contours of local SGS viscosity of SM and HM in the $x-z$ plane at $y^{+}=15$. It is much clearer to reveal the distribution of SGS viscosity in the $x$ - $z$ plane. In the laminar region, the SGS viscosity of SM has a fairly large value and the value of HM tends to zero, which is consistent with the conclusion from Fig. 2(a). In the transitional region, the spatial distribution of the SGS viscosity of HM also exhibits a rational intermittency.

From the a priori test, we obtain that the Smagorinsky model can generate unreasonable SGS dissipation in the laminar region (where $C_{s}$ should be zero), and the transition process will be slowed down and even not occur at all. For HM, the amount of SGS dissipation is negligible and the development of linear disturbances can be maintained before the occurrence of transition. To verify the validity in the simulation of transitional flows with the helicity model, an a posteriori test should be verified in the representative transitional flows.

\section{A POSTERIORI TEST}

\section{A. The numerical method}

In this section, we will give an a posteriori test of several SGS models with a supersonic spatially developing boundary layer ${ }^{23}$ with a free-stream Mach number of 2.25 and a free-stream unit

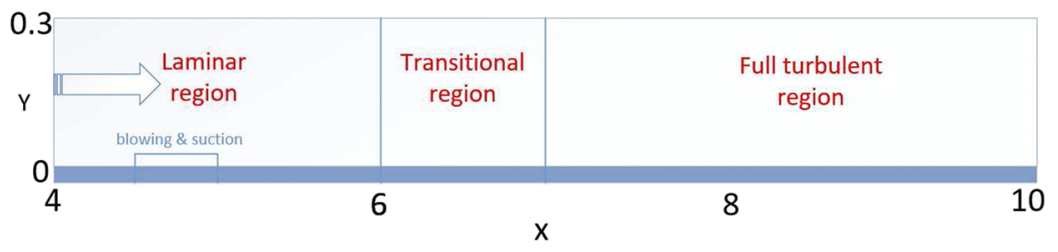

FIG. 1. Sketch of the computational domain for simulation. 
Reynolds number (Re/in.) of 635000 , a free-stream temperature $(T)$ of $169.44 \mathrm{~K}$, and an adiabatic wall temperature of $322 \mathrm{~K}$. We also give an $a$ posteriori test of several SGS models with supersonic flow over a $24^{\circ}$ compression ramp $^{24}$ with a free-stream Mach number of 2.9 and Reynolds number $\left(R e_{\theta}\right)$ of 2300 .

In the $a$ posteriori test, the governing equations (1)-(4) for LES of compressible flow are solved by using a high precision nondimensional finite difference solver in Cartesian coordinates, which is named Opencfd-SC and developed by our group for the compressible flows. ${ }^{25-27}$ The free-stream velocity, free-stream density, and unit length of mesh are selected as the characteristic velocity, characteristic density, and characteristic length. A sixth-order central difference scheme is employed for the discretization of the convective terms and the viscous terms. The discretized equations are temporally integrated using an explicit third-order Runge-Kutta scheme. In the case of supersonic flow over a compression ramp, in order to capture shock wave accurately, we use the shock-capturing methodology based on adaptive spatial filtering for high-order nonlinear computations in the corner region. ${ }^{2}$

In the tests, the main results for comparison from LES are derived by using five SGS models, namely, constant coefficient Smagorinsky model (CSM), dynamic Smagorinsky model (DSM), constant coefficient helicity model (CHM), dynamic helicity model (DHM), and wall-adapting local eddy-viscosity (WALE) model. ${ }^{4}$

The SGS viscosity of the WALE model is as follows:

$$
\mu_{W A L E}^{s g s}=\frac{\bar{\rho} C_{w} \Delta^{2}\left(\mathrm{~S}_{i j}^{d} \mathrm{~S}_{i j}^{d}\right)^{3 / 2}}{\left(\tilde{S}_{i j} \tilde{S}_{i j}\right)^{5 / 2}+\left(\mathrm{S}_{i j}^{d} S_{i j}^{d}\right)^{5 / 4}},
$$

with $C_{w} \approx 10.6 C_{s}^{2}$ and $S_{i j}^{d}$ being the traceless symmetric part of the square of the velocity gradient tensor,

$$
\mathrm{S}_{i j}^{d}=\frac{1}{2}\left(\frac{\partial \tilde{u}_{i}}{\partial x_{l}} \frac{\partial \tilde{u}_{l}}{\partial x_{j}}+\frac{\partial \tilde{u}_{j}}{\partial x_{l}} \frac{\partial \tilde{u}_{l}}{\partial x_{i}}\right)-\frac{1}{3} \delta_{i j} \frac{\partial \tilde{u}_{m}}{\partial x_{l}} \frac{\partial \tilde{u}_{l}}{\partial x_{m}} .
$$

Considering the selected cases in this paper, we ignore the isotropic part of the SGS tensor $\tau_{k k}^{s g s}$, the $C_{r}$ can be the determined empirically based on its expression in incompressible turbulence, ${ }^{2}$ and $C_{r} \approx 0.01$ here. The heat flux models of these models are all constant coefficient heat flux models (6), and $P r_{t}=0.9$.

In the simulation of wall-bound flows, the SGS viscosity of CSM and CHM will be constrained by the Van Driest damping function ${ }^{2}$ near the wall, and the form is shown as follows:

$$
D=\left[1-\exp \left(-\frac{y^{+}}{25}\right)\right]^{2} .
$$

\section{B. The results of supersonic transition and turbulent flat-plate boundary layer}

The computational domain for this case is the same as the DNS Settings in Sec. III; the grids used for DNS and LES of the supersonic spatially developing boundary layer are shown in Table I. DNS and LES use $2.8 \times 10^{8}$ and $1.3 \times 10^{7}$ grid points, respectively. The reliability verification of the DNS data can be found in our previous $\operatorname{article}^{30}$ and the grid sensitivity study is proved in Appendix B. In this simulation, we apply a weak blowing and suction perturbation
TABLE I. Characteristics of the computational grids for simulations of transitional flow.

\begin{tabular}{lcc}
\hline \hline Grid & $\Delta x^{+} \times \Delta y^{+} \times \Delta z^{+}$ & $N_{x} \times N_{y} \times N_{z}$ \\
\hline DNS & $6.02 \times 0.58 \times 5.47$ & $10900 \times 90 \times 320$ \\
LES & $40.1 \times 0.58 \times 17.5$ & $1500 \times 90 \times 100$ \\
\hline
\end{tabular}

to trigger the natural-type transition in the boundary layer of the flat plate.

Figures 3(a) and 3(b) show the distributions of the skin-friction coefficient $\left[C_{f}=\tau_{w} /\left(\rho_{\infty} U_{\infty}^{2} / 2\right)\right]$ along the streamwise direction as a function of the Reynolds number based on the conditions at the outer edge of the boundary layer $R e_{x}$. Figure 3 (a) shows the distribution of skin-fraction coefficient compared with the theoretical value and DNS result of Pirozzoli et al. ${ }^{23}$ The theoretical value predicted by the Blasius turbulence equation is based on the momentous thickness and Van Driest transform. It can be seen that in the fullturbulence region $\left(R e_{x}>5 \times 10^{-6}\right)$, the present DNS result is in good agreement with the theoretical value and that of Pirozzoli et al. Compared with the DNS result of Pirozzoli et al., the onset of transition in the present DNS is somewhat delayed which is caused by the different type of transition. Pirozzoli et al. adopted the bypass-type transition with large disturbance to get a longer turbulence region. On the contrary, in order to test the application of the helicity model in
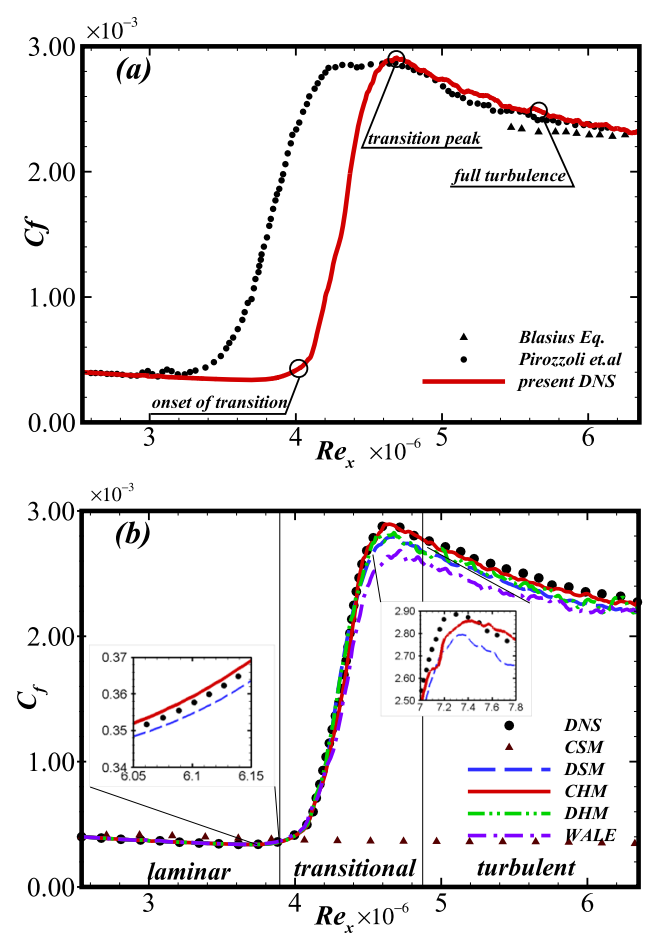

FIG. 3. Distributions of the skin-friction coefficient along the streamwise direction as a function of the Reynolds number based on the conditions at the outer edge of the boundary layer $R e_{x}$. (a) The present DNS compared with the theoretical value and the result of Pirozzoli et al..$^{23}$ and (b) the results of SGS models compared to DNS data. 
the transition problem, the natural-type transition with small disturbance is adopted in this paper. Figure 3(b) shows the distribution of the skin-fraction coefficient of the flat plate for five SGS models compared to DNS. The skin-friction of the DNS (black points) increases quickly when the boundary layer transition begins at approximately $R e_{x}>4 \times 10^{-6}$, reaches its maximum at approximately $R e_{x}>4.6$ $\times 10^{-6}$, and then gradually approaches the steady turbulent value as the boundary layer flow develops into full developed turbulence. Apparently, CSM (delta) cannot simulate transition from Fig. 3(b). Owing to the surplus SGS dissipation produced by CSM in the laminar region, the disturbances will decay quickly, which causes the flow not to transition as expected. In the meanwhile, CHM (solid) can predict the point of transition and transition peak accurately as explained in the previous part, and it can make HM differentiate laminar flow and turbulent flow automatically. For WALE (dasheddotted line), the "point" of transition can also be predicted, but the skin-friction of $R e_{x}>4.6 \times 10^{-6}$ is unsatisfactorily predicted. When the dynamic procedure is used, the SGS viscosity is automatically determined in the laminar and transitional regions. Both DSM (long-dashed line) and DHM (dashed-double-dotted line) predict the "point" of transition well. In summary, the skin-friction coefficient predicted by CHM is more consistent with the DNS data than other SGS models.

The distribution of the Van Driest transformed velocity $\left(U_{v d}\right.$ $\left.=\int_{0}^{U} \sqrt{\rho / \rho_{w}} d U\right)$ as a function of $y^{+}$in the full turbulent region $\left(R e_{x}=5.58 \times 10^{-6}\right)$ for these SGS models and DNS is shown in Fig. 4 . The dimensionless parameter $u_{\tau}$ adapted in Fig. 4(a) is calculated
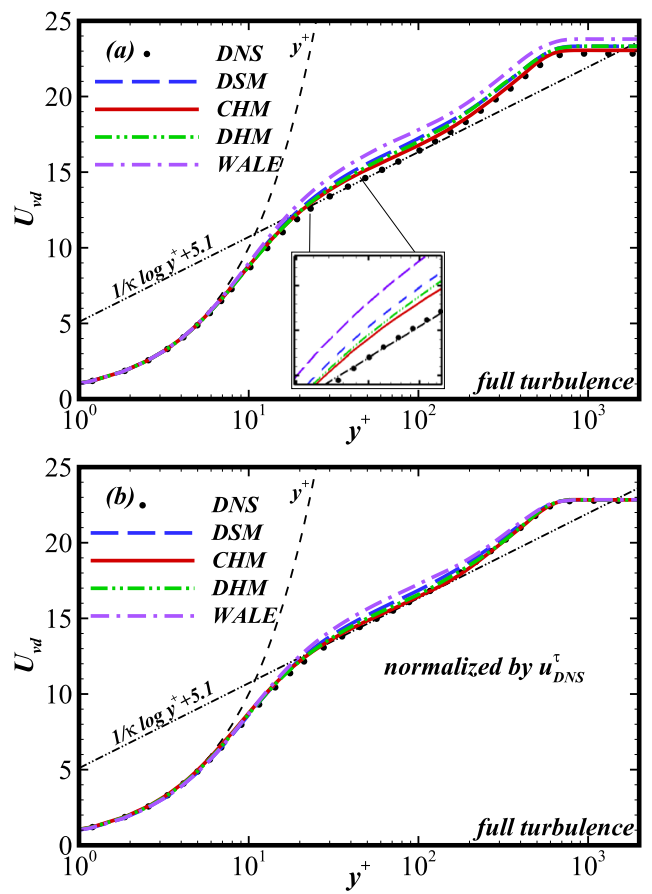

FIG. 4. Distribution of Van Driest transformed mean streamwise velocity in the full turbulence region, $R e_{x}=5.58 \times 10^{-6}$. (a) Dimensionless parameter $u_{\tau}$ is computed from LES data and (b) dimensionless parameter $u_{\tau}$ is computed from DNS data. from LES data; it can be seen that the profile from DNS is perfectly matched with a combination of the wall law and the logarithm law. Also, it can be easily seen that the profile from CHM is more closer to the DNS profile than that from other SGS models. More specifically,
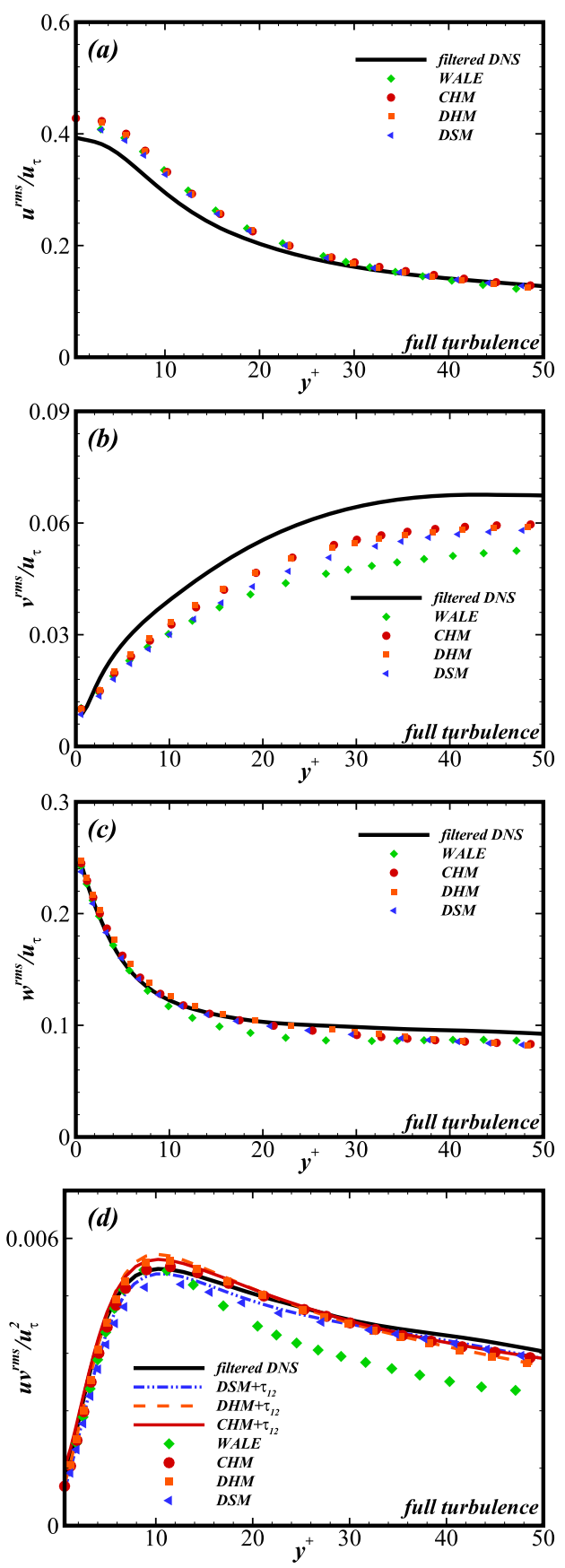

FIG. 5. Profiles of turbulence intensities normalized by friction velocity $u_{\tau}$ vs $y^{+}$in the full turbulence region, $R e_{x}=5.58 \times 10^{-6}$ : (a) streamwise turbulence intensity $U^{r m s}$, (b) normalwise turbulence intensity $V^{r m s}$, (c) spanwise turbulence intensity $W^{r m s}$, and (d) turbulence intensity UV ${ }^{r m s}$ 
in the viscous sublayer and the buffer region $\left(y^{+}<30\right)$, all models correspond well with the DNS data, and the velocity varies linearly with $y^{+}$in the domain of $y^{+}<6$ as expected. In the lag-law region $\left(20<y^{+}<100\right)$, most of the SGS models have a slight overestimation of $U_{v d}$; however, the result of CHM has obvious advantages in contrast with WALE, DSM, etc. In the outer part of the boundary layer, the profile of CHM collapses perfectly with the DNS data, which is more competitive than other models. The dimensionless parameter $u_{\tau}$ adapted in Fig. 4(b) is calculated from DNS; in the outer part of the boundary layer, all the results of the models fit tightly on the DNS result. In the log-law region, except that the profile from CHM showed consistency with the DNS data, the performance of other models is slightly overestimated.

Figures 5(a)-5(c) show the profiles of the resolved turbulence intensities $\tilde{u}_{i}^{r m s}=\left\langle\left(\tilde{u}_{i}-\left\langle\tilde{u}_{i}\right\rangle\right)^{2}\right\rangle^{1 / 2}$ obtained from CHM, compared with the filtered DNS result and that of WALE, DSM, and DHM in the full turbulence region, $R e_{x}=5.58 \times 10^{-6}$. Figure 5 (a) displays the streamwise turbulence intensity $U^{r m s}$, and we can see that the $\mathrm{CHM}$ result shows good agreement with the DNS data, and other models perform similarly. Figure 5(b) shows the normalwise turbulence intensity $V^{r m s}$, and it can be seen that all the SGS models have poor performance, and the performance of CHM and DHM is better than that of other models and DHM is also better than DSM. Figure 5(c) shows the spanwise turbulence intensity $W^{r m s}$, and all results of four models are close to the DNS result together. At the same time, the performance of the WALE model in the near-wall
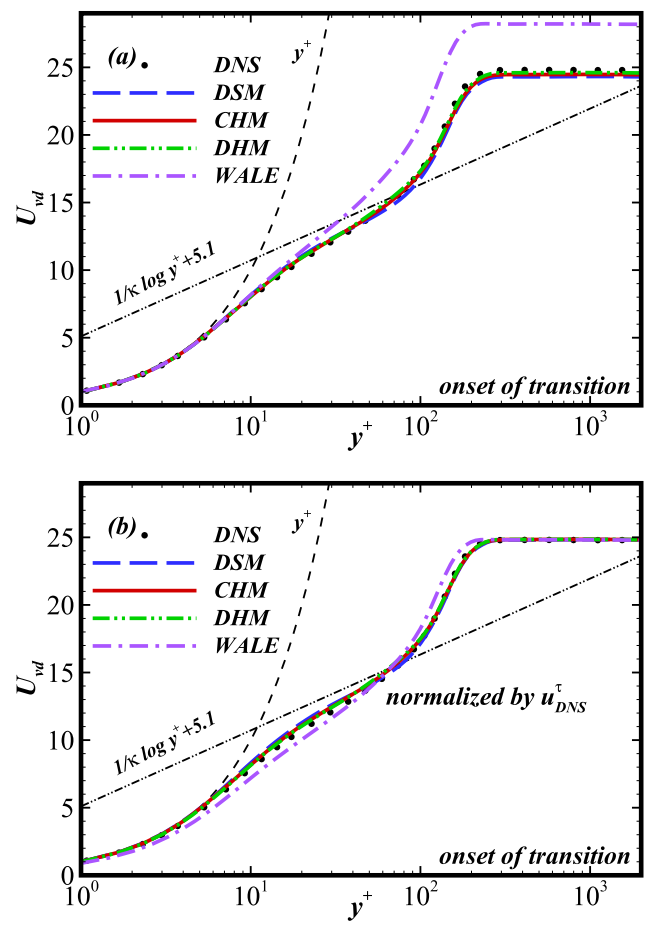

FIG. 6. Distribution of Van Driest transformed mean streamwise velocity at the early transitional stage, $R e_{x}=4.38 \times 10^{-6}$. (a) Dimensionless parameter $u_{\tau}$ is computed from LES data and (b) dimensionless parameter $u_{\tau}$ is computed from DNS data. region is better than that of other models. The performance of the two dynamic models is similar. In general, CHM performs well in the prediction of turbulence intensities.

Figure 5(d) shows the profiles of the resolved Reynolds stress $u v^{r m s}$ and the total Reynolds stress $\left(u v^{r m s}+\tau_{12}^{s g s}\right)$ from the LES result. It can be seen that in the near-wall region $\left(y^{+}<10\right)$ that all the models performed well, and the prediction of the resolved Reynolds stress by DHM and CHM is slightly higher than that by DNS. However, in the buffer region $\left(10<y^{+}<30\right)$, it can be seen that WALE can no longer fit the DNS data. This means that the wall adaptive behavior of WALE can indeed make the model perform well in the near-wall region, while $\mathrm{CHM}$ can simulate the behavior of the near-wall region after adapting the Van Driest damping function. In the prediction of the total Reynolds stress, the prediction of DHM and CHM is slightly higher than that of DSM.

In the early region of transition between $R e_{x}=4 \times 10^{-6}$ and $R e_{x}=5 \times 10^{-6}$, we also supply the comparison of these models in the transitional region.

Figures 6(a) and 6(b) show the mean velocity profiles of the location at the early transitional stage $\left(R e_{x}=4.38 \times 10^{-6}\right)$. The dimensionless parameter $u_{\tau}$ adapted in Fig. $6(\mathrm{a})$ is calculated from LES data; from Fig. 6(a), we can see a great difference with the results of the full turbulent region in Fig. 4, and the profiles of mean velocity have a sharp shift-down in the log-law region. At the same time, CHM can capture the typical flow character accurately at this location, and its profile is tightly consistent with the DNS data. On the contrary, WALE has an evident overestimation in the log-law region.
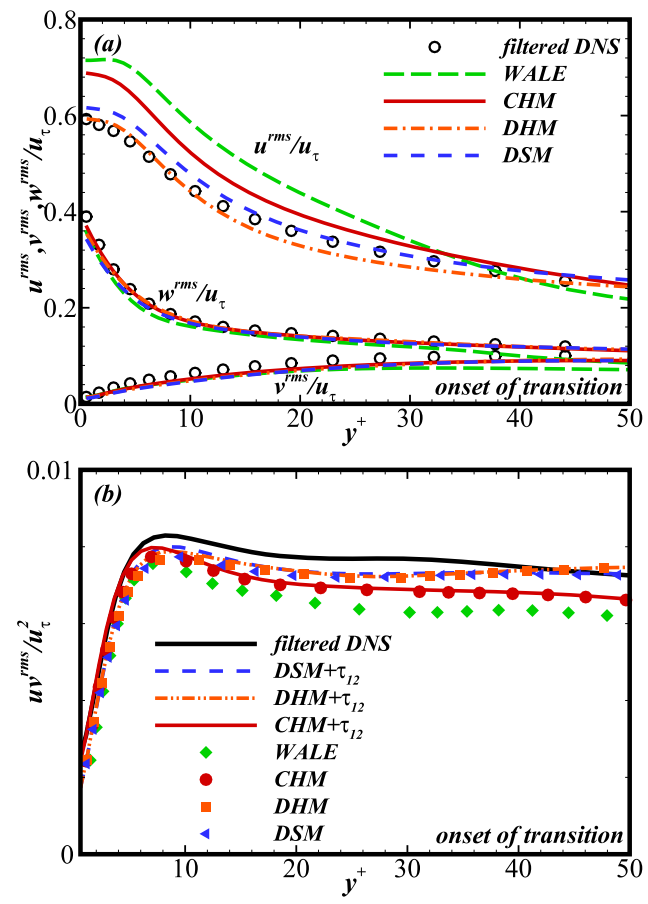

FIG. 7. Profiles of turbulence intensities and resolved Reynolds stress $u v^{r m s}$ normalized by friction velocity $u_{\tau}$ vs $y^{+}$at the early transitional stage, $R e_{X}=4.38$ $\times 10^{-6}$. (a) Turbulence intensities $u^{r m s}, v^{r m s}$, and $w^{r m s}$ and (b) resolved Reynolds stress $u v^{r m s}$ 
The dimensionless parameter $\boldsymbol{u}_{\tau}$ adapted in Fig. 6(b) is calculated from DNS, and in the outer part of the boundary layer, all the results of the models fit tightly on the DNS result. The velocity profile of WALE in the near-wall region and the log-law region is lower than the DNS data.

Figure 7(a) shows the profiles of the resolved turbulence intensities $\tilde{u}_{i}^{r m s}=\left\langle\left(\tilde{u}_{i}-\left\langle\tilde{u}_{i}\right\rangle\right)^{2}\right\rangle^{1 / 2}$ obtained from CHM, compared with the filtered DNS result and that of WALE, DSM, and DHM in the early transitional stage, $R e_{x}=4.38 \times 10^{-6}$. From Fig. 7(a), it can be seen that the performance of DHM is better than that of other models, and the performance of CHM, WALE, and DSM on $v^{r m s}$ and $w^{r m s}$ is in good agreement with the filtered DNS results. However, WALE overestimates the calculation of $u^{r m s}$. Figure 7(b) shows the profiles of the resolved Reynolds stress $u v^{r m s}$ and the total Reynolds stress $\left(u v^{r m s}+\tau_{12}^{s g s}\right)$ from the LES result. It can be seen that in the near-wall region $\left(y^{+}<10\right)$, CHM performs better than other models. In the buffer region $\left(10<y^{+}<30\right)$ and log-law region $\left(30<y^{+}<50\right)$, compared with the two dynamic models, CHM performs worse than dynamic models, but better than WALE. We think that the WALE model is based on the near-wall behavior in the turbulence region, and at the early transitional stage, the WALE model could not predict well the behavior of the near-wall. CHM may have accurately predicted the near-wall behavior at the early transitional stage due to the adoption of the appropriate Van Driest damping function. In the prediction of total Reynolds stress $\left(u v^{r m s}+\tau_{12}^{s g s}\right)$, the prediction results of $\mathrm{CHM}$ in the near-wall region are larger than that of the dynamic model.
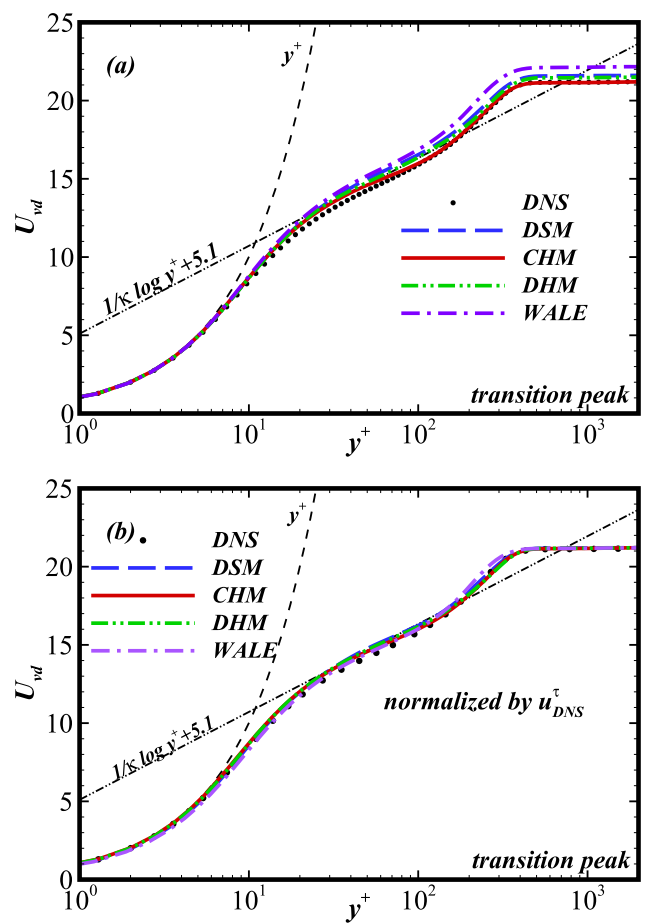

FIG. 8. Distribution of Van Driest transformed mean streamwise velocity at the transition peak, $R e_{x}=4.64 \times 10^{-6}$. (a) Dimensionless parameter $u_{\tau}$ is computed from LES data and (b) dimensionless parameter $u_{\tau}$ is computed from DNS data.
Figures 8(a) and 8(b) show the mean velocity profiles of the location at the transition peak $\left(R e_{x}=4.64 \times 10^{-6}\right)$. The dimensionless parameter $u_{\tau}$ adapted in Fig. 8(a) is calculated from LES data; from Fig. 8(a), we can see that the velocity profile is similar to the full turbulent region, and the WALE model is slightly different from DNS. The dimensionless parameter $u_{\tau}$ adapted in Fig. 8(b) is calculated from DNS, and it can be seen that the performance of WALE in the log-law region is better than that of other models after using $u_{\tau}^{D N S}$ as a dimensionless parameter, except for a little difference in the outer part of the boundary layer.

Figures 9(a) and 9(b) show the profiles of the resolved turbulence intensities $\tilde{u}_{i}^{r m s}=\left\langle\left(\tilde{u}_{i}-\left\langle\tilde{u}_{i}\right\rangle\right)^{2}\right\rangle^{1 / 2}$ obtained from CHM, WALE, DSM, DHM, and filtered DNS at the transition peak $\left(R e_{x}=4.64\right.$ $\times 10^{-6}$ ). As can be seen from Fig. 9(a), the performance of CHM is the best compared with other models, and the performance of DHM and DSM is roughly the same, while the performance of WALE is relatively poor compared with other models. Meanwhile, it can be seen from Fig. 9(b) that all the models seem to perform poorly, and the performance of CHM in the near-wall region $\left(y^{+}<10\right)$ is also as close as possible to the results of DNS. In the other region $\left(y^{+}>10\right)$, all the models are quite different from the results of DNS. The same as the prediction at the early transitional stage, the prediction result of CHM is slightly larger than that of the dynamic model in the prediction of the total Reynolds stress.

In general, the constant coefficient model (CHM) is superior to the dynamic model in the prediction of the turbulence region. The possible reason is that the constant coefficient model (CHM) is better than the dynamic model in the upstream region (the resolved
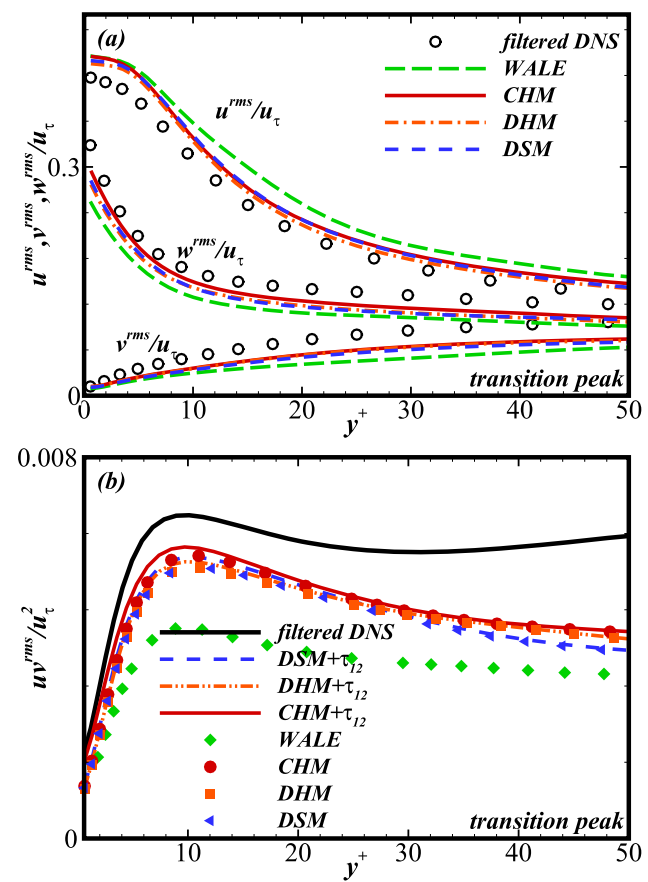

FIG. 9. Profiles of turbulence intensities and resolved Reynolds stress $u v^{r m s}$ normalized by friction velocity $u_{\tau}$ vs $y^{+}$transition peak, $R e_{x}=4.64 \times 10^{-6}$. (a) Turbulence intensities $u^{r m s}, v^{r m s}$, and $w^{r m s}$ and (b) resolved Reynolds stress $u v^{r m s}$. 
Reynolds stress at the early transitional stage and transition peak), which results in a better performance of CHM in the downstream region (turbulence region). There is a little difference between the two dynamic models, which can well simulate the near-wall behavior of the full turbulent region and accurately identify the occurrence of transition. WALE can simulate the near-wall behavior well in the full turbulent region, but it is not as good as other models at the early stage of transition and the transition peak. The issue with the WALE model may be related to the choice of the model coefficient and the numerical scheme. Here, the model coefficient is chosen as $C_{w} \approx 10.6 C_{s}^{2}$ in Ref. 31 , and we do not rectify the model coefficient according to our simulation.

In compressible flows, the prediction of temperature is also important for testing the SGS model. Here, we also give the results of temperature in transitional and turbulent regions.
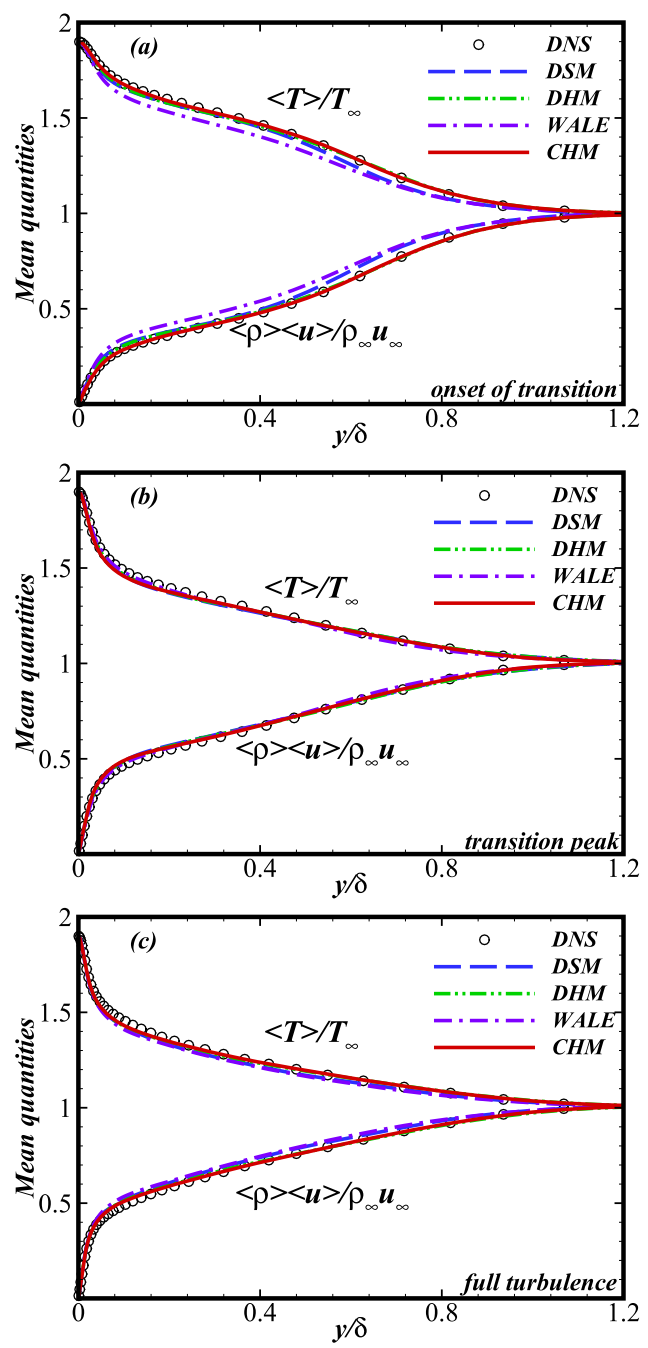

FIG. 10. Distributions of the time-averaged mean quantities as a function of $y / \delta$ at (a) early transitional stage, $R e_{x}=4.38 \times 10^{-6}$; (b) transition peak, $R e_{x}=4.64$ $\times 10^{-6}$; and (c) full turbulence, $\operatorname{Re}_{x}=5.58 \times 10^{-6}$ in inner scaling.
The distributions of the mean-averaged quantities as a function of $y / \delta$ in the location of different flow states are plotted in Figs. 10(a)-10(c), and the comparison with the DNS results reveals that the LES results are well reproduced in a large part of the boundary layer and the LES results exhibit a very little weakness in the $u$ and $T$ distributions around $y / \delta<0.3$. Overall, the model profiles conform well to the DNS data, and CHM seems to be the best in estimating averaged quantities, while WALE seems to be the worst, especially in the early transition stage.

The mean total temperature $\left\langle T_{t}\right\rangle$ is defined as

$$
\left\langle T_{t}\right\rangle=\langle T\rangle+\frac{1}{2} \frac{\gamma-1}{\gamma R}\left(\left\langle u_{i}\right\rangle^{2}+\left\langle u_{i}^{\prime} u_{i}^{\prime}\right\rangle\right)
$$

Figure 11(a)-11(c) show the distribution of normalized timeaveraged total temperature $T_{t} / T_{\infty}$ as a function of wall-normal
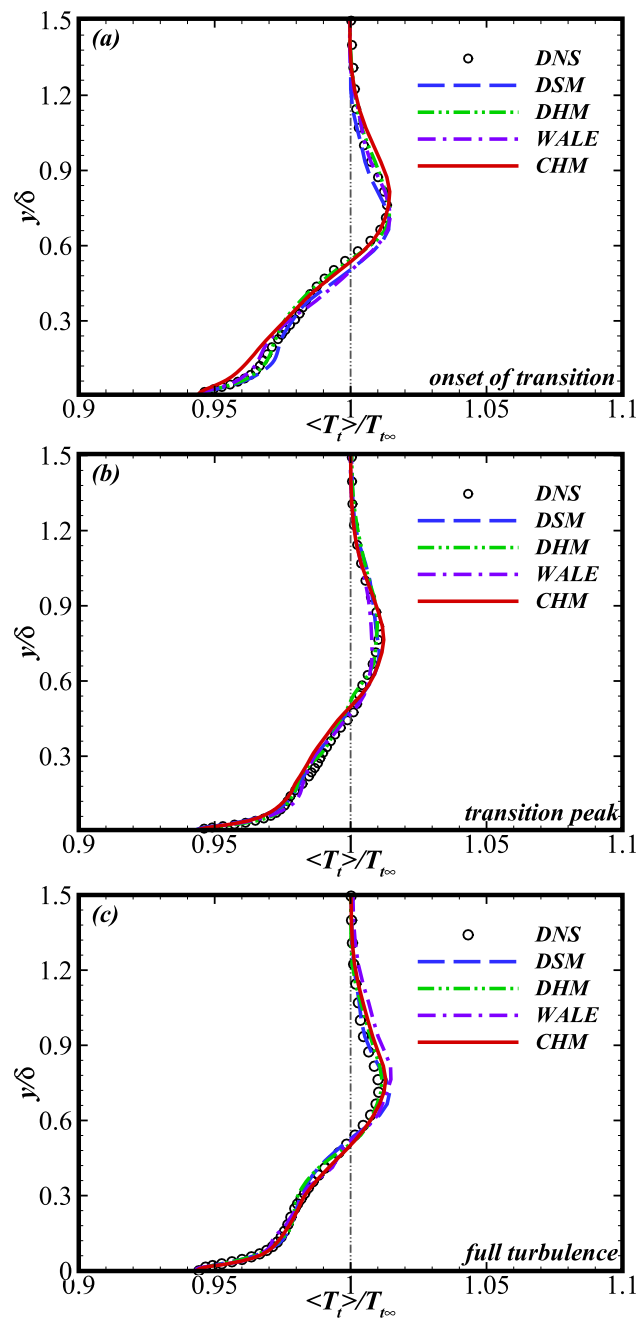

FIG. 11. Distribution of normalized time-averaged total temperature $T_{t} / T_{\infty}$ as a function of the wall-normal distance in outer scaling. (a) Early transitional stage, $R e_{x}=4.38 \times 10^{-6}$; (b) transition peak, $R e_{x}=4.64 \times 10^{-6}$; and (c) full turbulence, $\operatorname{Re}_{\mathrm{x}}=5.58 \times 10^{-6}$ 


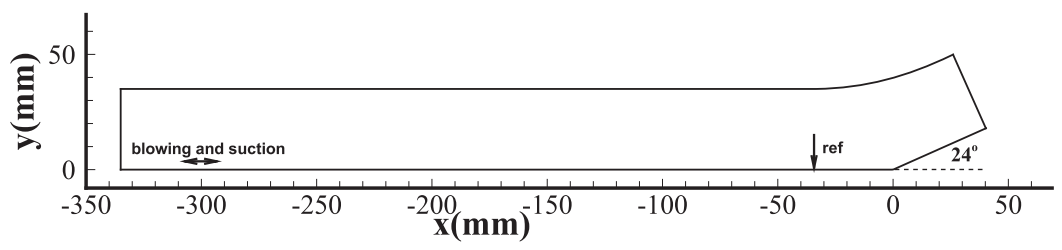

FIG. 12. Sketch of the computational domain of the compression ramp.

distance in outer scaling. As it can be seen in Fig. 11, the total temperature is not constant throughout the boundary layer. In fact, in the outer layer, the total temperature has been overshot, showing an overshoot that does not exceed $2 \%$ for all cases. This overshoot is expected to increase with positive heat flux, namely, the heated wall. This figure reflects the correlation between the SGS heat flux model and turbulent Prandtl number. Based on the figure, we could infer that the simple SGS heat flux model is not advantageous.

\section{The results of supersonic flow over a $24^{\circ}$ compression ramp}

Figure 12 shows the schematic diagram of the computing case, and the supersonic flow over a $24^{\circ}$ compression ramp is chosen in this case, ${ }^{27,32}$ which is the same as the experimental model of Bookey et l. $^{33}$ and research of $\mathrm{Wu}$ and Martin. ${ }^{24}$ The computational domains are $0 \leq y \leq 35 \mathrm{~mm}$ in the wall-normal direction and $0 \leq z \leq 14 \mathrm{~mm}$ in the spanwise direction, and the stream domain is $-335 \leq x \leq 49.56 \mathrm{~mm}$. To trigger the bypass-type transition, we impose the blowing and suction perturbation on the wall in $-305 \leq x \leq-285 \mathrm{~mm}$. In this section, we supply two sets of grids, one is used by DNS, and the other one is used by LES. The grid parameters are listed in Table II. In the upstream flat-plate region $(-335 \leq x \leq-35 \mathrm{~mm})$, the downstream grid point is denser than the upstream grid point. In the corner region $(-35 \leq x \leq 49.56 \mathrm{~mm})$, the much smaller streamwise grid spacing is used to resolve the small scales of STBLI and separation flows. For the LES, the streamwise grid spacing is uniform.

Figure 13(a) shows the distribution of the skin friction coefficient $C_{f}$ for the results of LES and DNS. The reliability verification of the DNS data can be found in our previous article. ${ }^{34}$ This figure shows that $C_{f}$ has a drastic increase near the region $x=-200 \mathrm{~mm}$, which denotes the occurrence of transition, unlike natural transition on a flat plate in Sec. IV B; this transition type here is bypass transition apparently. From the figure, we can see that the HM can also predict well the transition process in the case of bypass-type transition. In the corner region $(-35 \leq x \leq 35)$, the skin friction coefficient $C_{f}$ goes down rapidly downstream and then shows a negative value, indicating that the separation occurs here. The skin friction coefficient $C_{f}$ goes up rapidly and shows positive value again at $x=0 \mathrm{~mm}$, indicating the reattachment of the flow. We can see that all

TABLE II. Characteristics of the computational grids for simulations of supersonic flow over a $24^{\circ}$ compression ramp.

\begin{tabular}{lccccc}
\hline \hline Case & Grid number & $\Delta x^{+}(x=-335)$ & $\Delta x^{+}(x \geq-35)$ & $\Delta y_{w}^{+}$ & $\Delta z^{+}$ \\
\hline DNS & $4000 \times 160 \times 200$ & 6.52 & 2.90 & 0.58 & 4.06 \\
LES & $540 \times 100 \times 40$ & 40.60 & 40.60 & 0.87 & 20.30 \\
\hline
\end{tabular}

LES results are close to the DNS data. In order to carefully compare the performances of each model in the separation flow, Fig. 13(b) shows the distribution of the skin friction coefficient $C_{f}$ in the corner region, where the horizontal coordinates are normalized by $\delta$ (the boundary-layer thickness). As can be seen from this figure, the size of the separation bubble calculated by DSM is obviously smaller than the DNS data, the separation bubble calculated by other models is in good agreement with DNS data, and WALE seems a little better than other SGS models (see Table III).
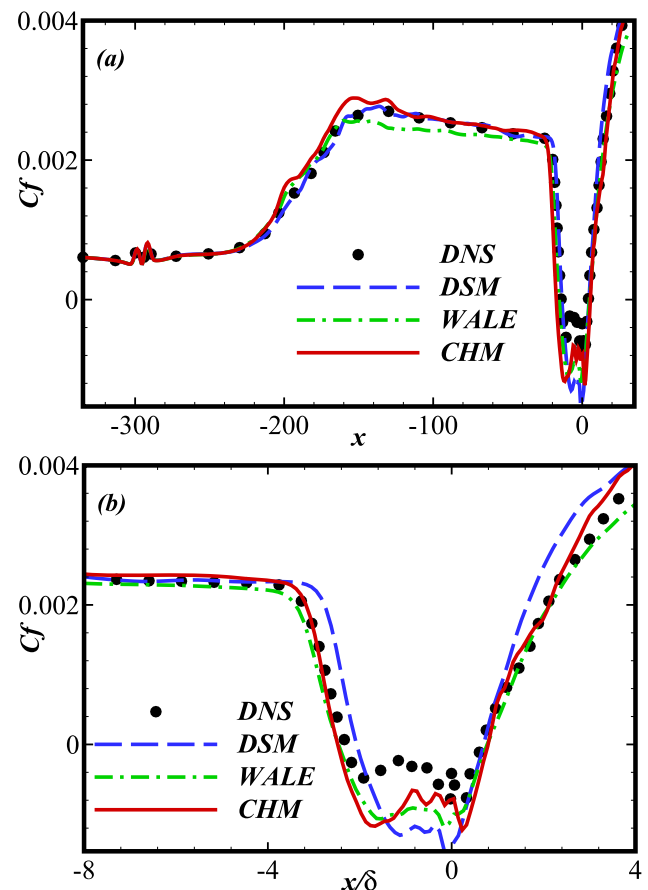

FIG. 13. (a) Distribution of the skin-friction coefficient along the streamwise direction and (b) distribution of the skin friction coefficient in the corner region, where the horizontal coordinates are normalized by $\delta$.

TABLE III. The bubble size predicted by models and their \% error compared to DNS.

\begin{tabular}{lcccc}
\hline \hline Case & $\begin{array}{c}\text { Separation } \\
\text { point }\end{array}$ & $\begin{array}{c}\text { Reattachment } \\
\text { point }\end{array}$ & $\begin{array}{c}\text { Separation } \\
\text { bubble }\end{array}$ & Error (\%) \\
\hline DNS & -2.31 & 0.67 & 2.98 & 0 \\
DSM & -2.07 & 0.70 & 2.77 & -7.0 \\
WALE & -2.47 & 0.78 & 3.25 & 9.0 \\
CHM & -2.48 & 0.79 & 3.27 & 9.7 \\
\hline \hline
\end{tabular}



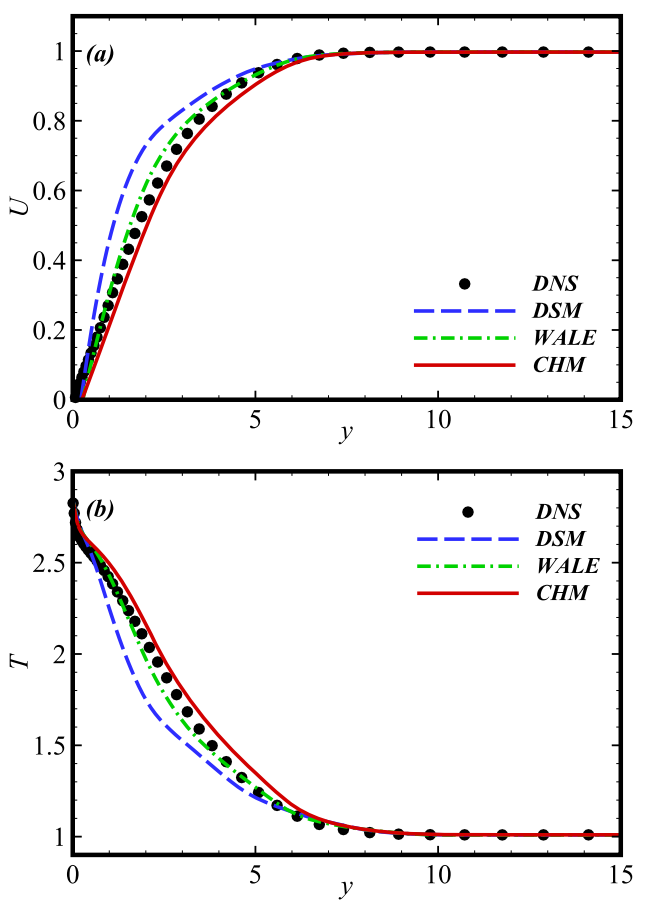

FIG. 14. (a) Distribution of streamwise velocity at $x / \delta=-1$ and (b) distribution of temperature at $x / \delta=-1$.
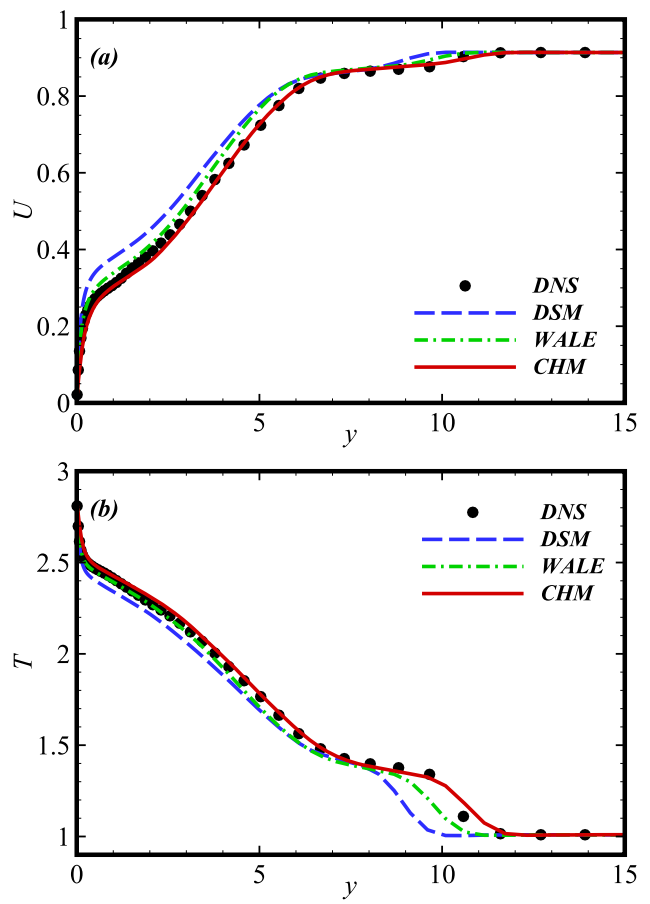

FIG. 15. (a) Distribution of streamwise velocity at $x / \delta=2$ and (b) distribution of temperature at $x / \delta=2$.
Figure 14 shows the distributions of streamwise velocity $U$ and temperature $T$ at $x / \delta=-1$. In this region, the flow is separated, and we can see that the profiles of streamwise velocity $U$ and temperature $T$ calculated by CHM are closer to the DNS data than those of other models. Figure 15 displays the distributions of streamwise velocity $U$ and temperature $T$ at $x / \delta=2$. Which is the location aflter the reattachment point. Shown in Figs. 15(a) and 15(b), CHM gives well the results in contrast to other models. From the simulation results in Figs. 14 and 15, we can infer that CHM can simulate the separation-reattachment process well in the region of the corner.

\section{CONCLUSIONS}

Based on the helicity model proposed in our previous work, we make some improvements, and a theoretical analysis for this model is supplied in this paper. From the analysis, we find that the helicity model can differentiate laminar and turbulent regions automatically in compressible flows. Using the DNS data of a supersonic spatially developing flat plate boundary layer with a free-stream Mach number of 2.25, we make an a priori test for the proposed model. The test results reveal that the helicity model unlike the traditional Smagorinsky model can provide a suitable SGS turbulent dissipation in laminar and transitional regions, which is the guarantee for development of disturbances in the laminar boundary layer and occurrence of transition. From theoretical analysis and the a priori test, we can also conclude that the helicity model with a constant coefficient has a special SGS viscosity, which is just like a switch to control the SGS model in different stages of the flows.

In the a posteriori test, we choose the natural-type transition case (flat plate boundary layer) and bypass-type transition (compression ramp) to verify the validity of the helicity model. In the flat plate boundary layer, we make tests of the distribution of the skin-friction coefficient, mean velocity profile, mean temperature profile, turbulence intensities, and some other mean quantities. The results show that the helicity model has obvious advantages among these compared models. In the flow over a compression ramp, the location of transition, mean velocity and temperature profiles at the separated and reattached points, and the location and size of the separation bubble can also be predicted well by the helicity model.

\section{ACKNOWLEDGMENTS}

This work was supported by the National Key Research and Development Program of China (Grant No. 2019YFA0405302 and 2016YFA0401200) and the NSFC Projects (Nos. 91852203, 91441103, 11372330 and 11472278), the Science Challenge Project (No. TZ2016001), and the Strategic Priority Research Program of Chinese Academy of Sciences (Grant Nos. XDA17030100 and XDC01000000). The authors thank the National Supercomputer Center in Tianjin (NSCC-TJ) and the National Supercomputer Center in Guangzhou (NSCC-GZ) for providing computer time.

\section{APPENDIX A: GALILEAN INVARIANCE}

It is well known for a long time that the Navier-Stokes equations are Galilean invariant. To keep the LES equations Galilean invariant, the SGS viscosity must be ensured Galilean invariant.

We define $\mathrm{E}=\left\{\mathbf{e}_{1}, \mathbf{e}_{2}, \mathbf{e}_{3}\right\}$ and $\mathrm{E}^{*}=\left\{\mathbf{e}_{1}^{*}, \mathbf{e}_{2}^{*}, \mathbf{e}_{3}^{*}\right\}$ as two unit orthonormal bases, and there exists an orthogonal transformations 
as follows;

$$
\mathbf{e}_{i}^{*}=C_{i j} \mathbf{e}_{j},
$$

where the matrix $C_{i j}$ is called the direction-cosine matrix and the repeated indexes satisfy the Einstein notation.

We write the Galilean transformation for two arbitrary coordinates as follows:

$$
x_{i}^{*} \mathbf{e}_{i}^{*}=x_{i} \mathbf{e}_{i}+U t+b,
$$

where $U_{i}$ is the relative velocity and $b_{i}$ is the relative distance at the initial time of two coordinates. Substituting relation (A1) into (A2) and considering any one term of the relation, we can easily get the following equation:

$$
x_{i}=-b_{i}-U_{i} t+C_{k i} x_{k}^{*}=-b_{i}-U_{i} t^{*}+C_{k i} x_{k}^{*} .
$$

Here, $t^{*}$ is the time of the relative coordinate and $t^{*}=t$. The derivative of both sides of relation (A2) with respect to time is as follows:

$$
v_{i}^{*} \mathbf{e}_{i}^{*}=U_{i}+v_{i} \mathbf{e}_{i}
$$

where $\mathbf{v}$ and $\mathbf{v}^{*}$ are the velocity of two coordinates. Substituting relation (A1) into (A4), we can get the following equation:

$$
v_{i}^{*} C_{i j} \mathbf{e}_{j}=\left(U_{i}+v_{i}\right) \mathbf{e}_{i} .
$$

Hence, the relative velocity can be expressed as follows:

$$
v_{i}^{*}=\left(v_{k}+U_{k}\right) C_{k i}^{T}=\left(v_{k}+U_{k}\right) C_{i k} .
$$

The velocity gradient can be expanded as follows:

$$
\begin{aligned}
\frac{\partial v_{i}^{*}}{\partial x_{j}^{*}} & =\frac{\partial v_{i}^{*}}{\partial x_{k}} \frac{\partial x_{k}}{\partial x_{j}^{*}} \\
& =C_{i m} C_{j k} \frac{\partial v_{m}}{\partial x_{k}} \\
& =C_{i m} \frac{\partial v_{m}}{\partial x_{k}} C_{k j}^{T} .
\end{aligned}
$$

Hence, the following equations can be easily deduced as follows:

$$
S_{i j}^{*}=C S_{i j} C^{T}, \quad|S|^{*}=|S| .
$$

So far, the Galilean invariance of the Smagorinsky model can be proved.

Similarly, the following equation exists for the gradient of vorticity:

$$
\frac{\partial \omega_{i}^{*}}{\partial x_{j}^{*}}=C \frac{\partial \omega_{i}}{\partial x_{j}} C^{T} \operatorname{det}(C),
$$

where det denotes the determinant of matrix $R$. So, we can prove that

$$
R_{i j}^{*}=\frac{1}{2}\left(\frac{\partial \omega_{i}^{*}}{\partial x_{j}^{*}}+\frac{\partial \omega_{j}^{*}}{\partial x_{i}^{*}}\right)=C R_{i j} C^{T} \operatorname{det}(C) .
$$

Hence,

$$
S_{s r}^{*}=\left|2 S_{i j}^{*} R_{i j}^{*}\right|=S_{s r} .
$$

So we can prove that the helicity model we proposed also satisfies the Galilean invariance.
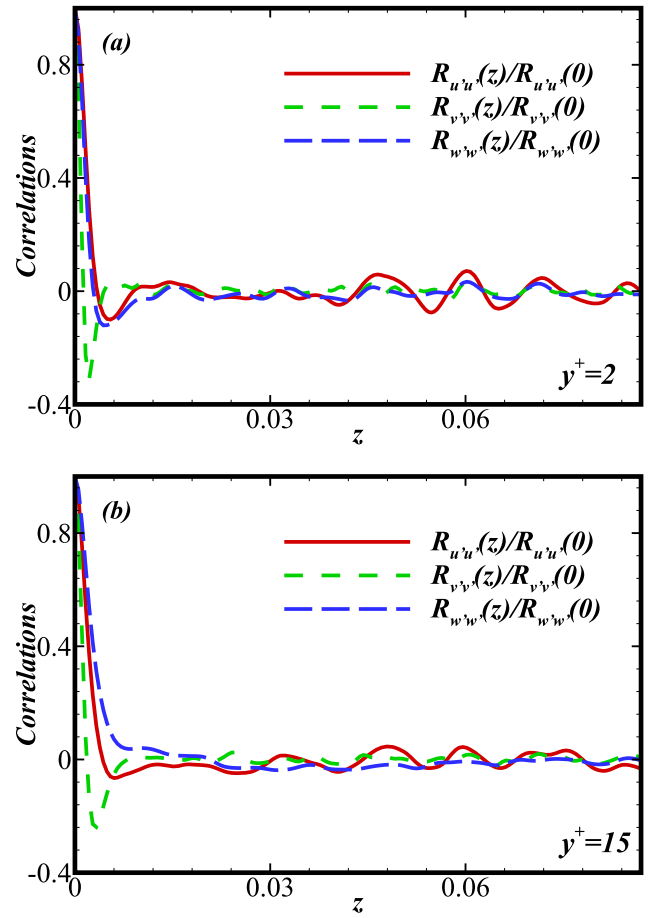

FIG. 16. Two-point correlation functions at (a) $y^{+}=2$ and (b) $y^{+}=15$

\section{APPENDIX B: GRID SENSITIVITY STUDY}

To ensure that the computational domain is sufficiently wide in the spanwise direction $Z$, the two-point correlation functions are given.

Figure 16 show the two-point correlation functions at $y^{+}=2$ and $y^{+}=15$; it can be seen that the distributions decrease to zero, which means that the domain is wide enough in the spanwise direction $Z$.

In Fig. 17, we give a comparison of the results of some models on a coarser grid (see Table IV). From the results, it can be seen that the performance of models on the coarse grid is worse than that on the fine grid, and the results obtained on the fine grid are also applicable to the coarse grid.

\section{APPENDIX C: THE DYNAMIC PROCEDURE}

In the dynamic procedure, the model's coefficient $C s$ is dynamically extracted from the resolved flow field. A test box-filter is denoted as $\widehat{(\cdot)}$, whose width is twice the grid-filter width. Then, we can get the model's coefficient $C s^{d}$ as

$$
C s^{d}=\frac{\left\langle M_{i j} L_{i j}^{d}\right\rangle}{\left\langle M_{k l} M_{k l}\right\rangle},
$$

where $M_{i j}=\alpha_{i j}-\widehat{\beta_{i j}}$ and $L_{i j}^{d}=L_{i j}-\frac{1}{3} L_{k k} \delta_{i j}$. Taking the dynamic Smagorinsky model as an example,

$$
L_{i j}=\widehat{\bar{\rho} \hat{\tilde{u}}_{i} \tilde{u}_{j}}-\frac{1}{\hat{\bar{\rho}}} \widehat{\bar{\rho}} \hat{\tilde{u}}_{i} \widehat{\rho} \widehat{\tilde{u}}_{j},
$$



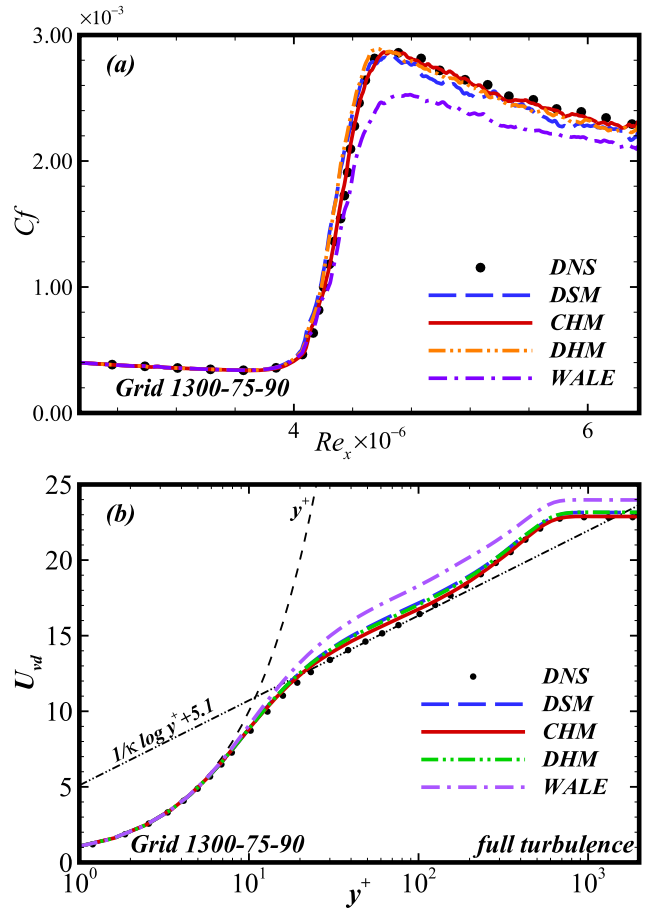

FIG. 17. (a) Distributions of the skin-friction coefficient along the streamwise direction as a function of the Reynolds number based on the conditions at the outer edge of the boundary layer, $R e_{x}$, and (b) distributions of Van Driest transformed mean streamwise velocity in the full turbulence region, $R e_{x}=5.58 \times 10^{-6}$.

TABLE IV. Characteristics of a coarser grid.

\begin{tabular}{lll}
\hline \hline Grid & $\Delta x^{+} \times \Delta y^{+} \times \Delta z^{+}$ & $N_{x} \times N_{y} \times N_{z}$ \\
\hline DNS & $6.02 \times 0.58 \times 5.47$ & $10900 \times 90 \times 320$ \\
LES & $44.8 \times 0.87 \times 18.9$ & $1300 \times 75 \times 90$ \\
\hline \hline
\end{tabular}

$$
\alpha_{i j}=-2(2 \Delta)^{2} \hat{\hat{\rho}}|\hat{\tilde{S}}|\left(\hat{\tilde{S}}_{i j}-\frac{1}{3} \delta_{i j} \hat{\tilde{S}}_{k k}\right)
$$

and

$$
\beta_{i j}=-2 \Delta^{2} \bar{\rho}|\tilde{S}|\left(\tilde{S}_{i j}-\frac{1}{3} \delta_{i j} \tilde{S}_{k k}\right) .
$$

Meanwhile, the model's coefficient $C s^{d}$ is guaranteed to be of no negative value.

\section{REFERENCES}

${ }^{1}$ J. Smagorinsky, "General circulation experiments with the primitive equations: I. The basic experiment," Mon. Weather Rev. 91, 99-164 (1963).

${ }^{2} \mathrm{O}$. Métais and M. Lesieur, "Spectral large-eddy simulation of isotropic and stably stratified turbulence," J. Fluid Mech. 239, 157-194 (1992).

${ }^{3}$ G. Cui, H. Zhou, Z. Zhang, and L. Shao, "A new dynamic subgrid eddy viscosity model with application to turbulent channel flow," Phys. Fluids 16, 2835-2842 (2004).
${ }^{4}$ F. Nicoud and F. Ducros, "Subgrid-scale stress modelling based on the square of the velocity gradient tensor," Flow, Turbul. Combust. 62, 183-200 (1999).

${ }^{5} \mathrm{~A}$. Vreman, "An eddy-viscosity subgrid-scale model for turbulent shear flow: Algebraic theory and applications," Phys. Fluids 16, 3670-3681 (2004).

${ }^{6}$ L. Marstorp, G. Brethouwer, O. Grundestam, and A. V. Johansson, "Explicit algebraic subgrid stress models with application to rotating channel flow," J. Fluid Mech. 639, 403-432 (2009).

${ }^{7}$ A. Rasam, S. Wallin, G. Brethouwer, and A. V. Johansson, "Large eddy simulation of channel flow with and without periodic constrictions using the explicit algebraic subgrid-scale model," J. Turbul. 15, 752-775 (2014).

${ }^{8}$ W. Rozema, H. J. Bae, P. Moin, and R. Verstappen, "Minimum-dissipation models for large-eddy simulation," Phys. Fluids 27, 085107 (2015).

${ }^{9}$ M. Germano, U. Piomelli, P. Moin, and W. H. Cabot, "A dynamic subgrid-scale eddy viscosity model,” Phys. Fluids A 3, 1760-1765 (1991).

${ }^{10} \mathrm{C}$. Yu, Z. Xiao, and X. Li, "Scale-adaptive subgrid-scale modelling for large-eddy simulation of turbulent flows," Phys. Fluids 29, 035101 (2017).

${ }^{11}$ C. Yu, Z. Xiao, and X. Li, "Dynamic optimization methodology based on subgrid-scale dissipation for large eddy simulation," Phys. Fluids 28, 015113 (2016).

${ }^{12}$ F. Porté-Agel, C. Meneveau, and M. B. Parlange, "A scale-dependent dynamic model for large-eddy simulation: Application to a neutral atmospheric boundary layer," J. Fluid Mech. 415, 261-284 (2000).

${ }^{13}$ C. Meneveau and T. S. Lund, "The dynamic Smagorinsky model and scaledependent coefficients in the viscous range of turbulence," Phys. Fluids 9, 3932-3934 (1997).

${ }^{14}$ A. Scotti, C. Meneveau, and M. Fatica, "Dynamic Smagorinsky model on anisotropic grids," Phys. Fluids 9, 1856-1858 (1997).

${ }^{15}$ A. Yoshizawa, "A statistically-derived subgrid model for the large-eddy simulation of turbulence," Phys. Fluids 25, 1532-1538 (1982).

${ }^{16} \mathrm{~T}$. Sayadi and P. Moin, "Large eddy simulation of controlled transition to turbulence," Phys. Fluids 24, 114103 (2012).

${ }^{17} \mathrm{~K}$. Horiuti, "On the use of SGS modeling in the simulation of transition in plane channel flow," J. Phys. Soc. Jpn. 55, 1528-1541 (1986).

${ }^{18}$ F. Ducros, P. Comte, and M. Lesieur, "Large-eddy simulation of transition to turbulence in a boundary layer developing spatially over a flat plate," J. Fluid Mech. 326, 1-36 (1996).

${ }^{19} \mathrm{X}$. Huai, R. D. Joslin, and U. Piomelli, "Large-eddy simulation of transition to turbulence in boundary layers," Theor. Comput. Fluid Dyn. 9, 149-163 (1997).

${ }^{20} \mathrm{C}$. Yu, R. Hong, Z. Xiao, and S. Chen, "Subgrid-scale eddy viscosity model for helical turbulence," Phys. Fluids 25, 095101 (2013).

${ }^{21}$ M. P. Martin, U. Piomelli, and G. V. Candler, "Subgrid-scale models for compressible large-eddy simulations,” Theor. Comput. Fluid Dyn. 13, 361-376 (2000).

${ }^{22}$ U. Piomelli, T. A. Zang, C. G. Speziale, and M. Y. Hussaini, "On the largeeddy simulation of transitional wall-bounded flows," Phys. Fluids A 2, 257-265 (1990).

${ }^{23}$ S. Pirozzoli, F. Grasso, and T. Gatski, "Direct numerical simulation and analysis of a spatially evolving supersonic turbulent boundary layer at $M=2.25$," Phys. Fluids 16, 530-545 (2004).

${ }^{24} \mathrm{M}$. Wu and M. P. Martin, "Direct numerical simulation of supersonic turbulent boundary layer over a compression ramp," AIAA J. 45, 879-889 (2007).

${ }^{25} \mathrm{X}$. Li, D. Fu, and Y. Ma, "Direct numerical simulation of hypersonic boundary layer transition over a blunt cone with a small angle of attack," Phys. Fluids 22, 025105 (2010).

${ }^{26}$ Y.-S. Zhang, W.-T. Bi, F. Hussain, and Z.-S. She, "A generalized Reynolds analogy for compressible wall-bounded turbulent flows," J. Fluid Mech. 739, 392-420 (2014).

${ }^{27}$ F. Tong, X. Li, Y. Duan, and C. Yu, "Direct numerical simulation of supersonic turbulent boundary layer subjected to a curved compression ramp," Phys. Fluids 29, 125101 (2017).

${ }^{28}$ C. Bogey, N. De Cacqueray, and C. Bailly, "A shock-capturing methodology based on adaptative spatial filtering for high-order non-linear computations," J. Comput. Phys. 228, 1447-1465 (2009).

${ }^{29}$ E. V. Driest, "On turbulent flow near a wall," J. Aeronaut. Sci. 23, 1007-1011 (1956). 
${ }^{30}$ L. Xin-Liang, F. De-Xun, M. Yan-Wen, and G. Hui, "Acoustic calculation for supersonic turbulent boundary layer flow," Chin. Phys. Lett. 26, 094701 (2009).

${ }^{31}$ E. Garnier, N. Adams, and P. Sagaut, Large Eddy Simulation for Compressible Flows (Springer Science \& Business Media, 2009).

${ }^{32} \mathrm{~K}$. M. Porter and J. Poggie, "Selective upstream influence on the unsteadiness of a separated turbulent compression ramp flow," Phys. Fluids 31, 016104 (2019).
${ }^{33}$ P. Bookey, C. Wyckham, A. Smits, and P. Martin, "New experimental data of STBLI at DNS/LES accessible Reynolds numbers," in 43rd AIAA Aerospace Sciences Meeting and Exhibit (AIAA, 2005), p. 309.

${ }^{34}$ X.-K. Zhu, C.-P. Yu, F.-L. Tong, and X.-L. Li, "Numerical study on wall temperature effects on shock wave/turbulent boundary-layer interaction," AIAA J. 55, 131-140 (2016). 